\title{
Exploring Post-Activation Conformational Changes in Pigeon Cryptochrome 4 (Supplementary Material)
}

\author{
Fabian Schuhmann, ${ }^{\dagger}$ Daniel R. Kattnig, ${ }^{\ddagger}$ and Ilia A. Solov'yov ${ }^{*, \dagger}$ \\ $\dagger$ Department of Physics, Carl von Ossietzky Universät Oldenburg \\ Carl-von-Ossietzky Str. 9-11, 26129 Oldenburg, Germany \\ $\ddagger$ University of Exeter, Stocker Rd., Exeter, EX4 4QD, U.K. \\ Living Systems Institute and Department of Physics \\ E-mail: ilia.solovyov@uni-oldenburg.de
}




\section{Protein stability}

The solvated structure of ClCry4 was extensively equilibrated to ensure protein stability. Figure S1 shows the RMSD computed for the protein in its DS and RPD state for all simulations after equilibration. The first 375 ns of the production simulation were not considered for analysis for all simulation sets to allow the RPD states to equilibrate. During the simulation time interval from $375 \mathrm{~ns}$ to $1000 \mathrm{~ns}$, the DS and RPD structures have an

RMSD smaller than $4 \AA$ that indicates rather modest internal rearrangements (if any). The RMSD time evolution allows to conclude about protein stability but does not allow a direct comparison of internal dynamics in the two considered structures. Furthermore, RMSD plots for the ClCry4 structure omitting residues 228-244 were included to judge how much of the RMSD fluctuations in the top plots of Fig. S1 can be attributed to this intrinsically unordered region. The results are most clear when comparing the RMSD plots for RPD1 and RPD2 in Fig. S1. One notes that the climb in RMSD is significantly smaller for RPD1 or even completely absent for RPD2. Furthermore, the increase in RMSD at the end of the DS1 simulation can be attributed to rearrangements in residues 228-244, leading to further closing of the gate controling the passage towards the FAD binding pocket. This becomes clear after the analysis of the DS3/RPD3 simulation (see main text) where the creation of a radical pair (RPD3) does not cause a clear rearrangement within a simulated microsecond time interval. For both simulations sets 1 and 2, the rearrangements of the RPD states, evidenced by the increasing RMSD consistently correspond to the motion of the phosphatebinding domain residues in one direction, move away from the respective position in the dark state structures, see Fig. 6 and Fig. 7 . 

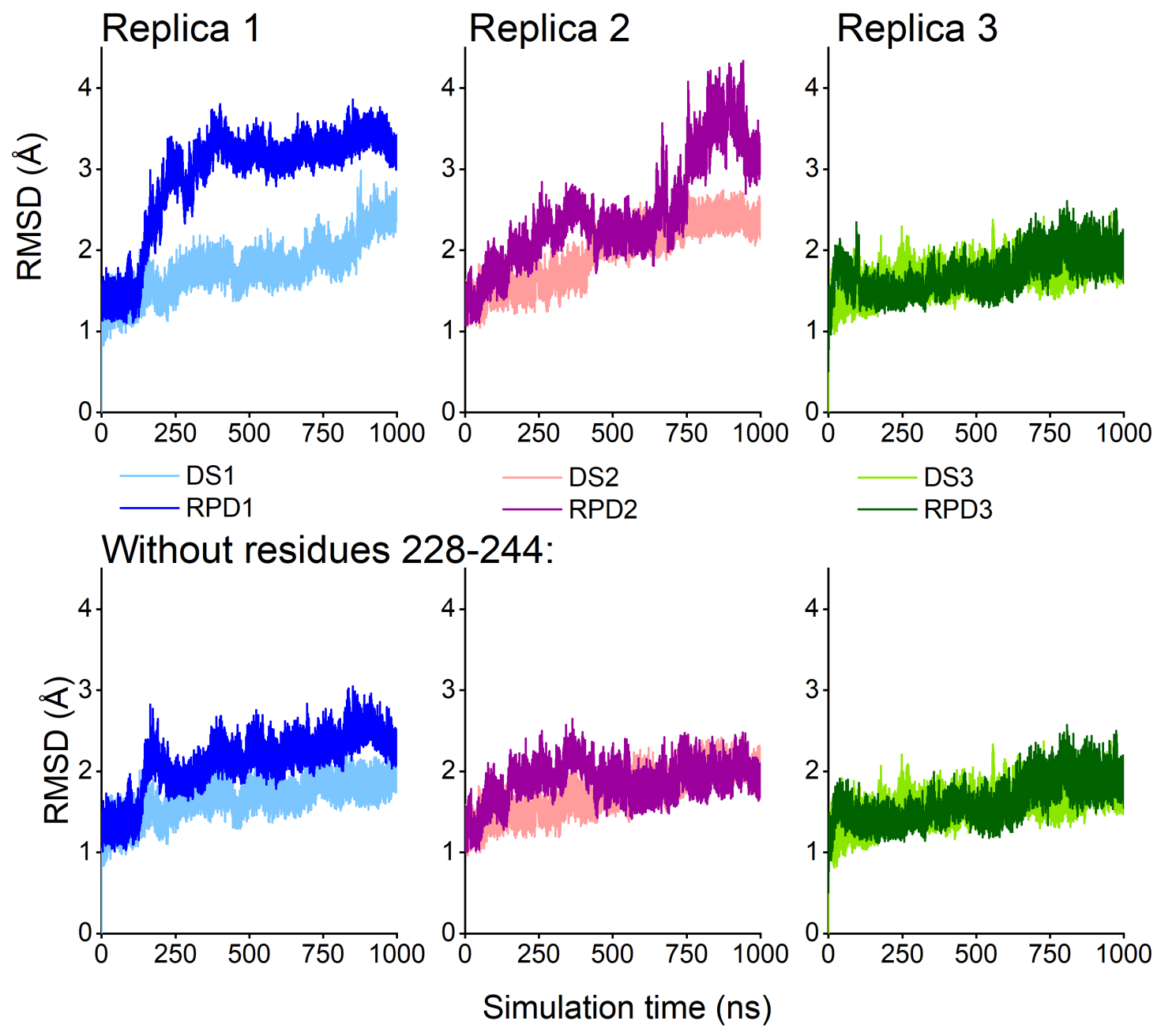

Figure S1: Time evolution of the root mean square displacement (RMSD) computed for the backbone of the entire ClCry4 (top row) and the backbone excluding residues 228-244 (bottom row). Results are shown for the three sets of replica simulations (DS1/RPD1, DS2/RPD2, DS3/RPD3) as indicated by colors. RMSD was computed by aligning each snapshot relative to the first MD snapshot as a reference structure. The climb in the DS1 RMSD close to the end of the simulation can be attributed to movement of residues 228-244 that causes additional stability of the phosphate-binding loop as seen in the DS3 simulation. To confirm that the change in RMSD is primarily arising due to that region, the RMSD was computed once more for every simulation omitting residues 228-244. One notices that the climb in the RMSD of RPD1 and RPD2 is only present if the loop is considered. Furthermore, there is no marked difference in the behavior of the RMSD over time for simulations DS3 and RPD3. 


\section{The principal component analysis and distance matrix method}

An earlier study ${ }^{1}$ proposed an analysis to show conformational changes in the ErCry4a homology model when the protein is converted into the radical pair state. A similar recipe to show the changes in ClCry4 was applied. All the analysis relied on internal dynamics of the protein, i.e., required the overall motion of the protein to be removed from the DS simulation and aligning the snapshots of the RPD simulations to its corresponding average DS structure. The alignment scheme is the same as the one described in detail earlier, ${ }^{1}$ in which all MD snapshots were iteratively aligned to an average structure until the changes were insignificant given a threshold.

\section{Distance Matrix}

We define the distance matrix $A$ with entries $a_{i j}$ as

$$
a_{i j}=\left\langle\left\|\vec{r}_{i}^{D S}-\vec{r}_{j}^{D S}\right\|\right\rangle-\left\langle\left\|\vec{r}_{i}^{R P D}-\vec{r}_{j}^{R P D}\right\|\right\rangle
$$

where $\|\cdot\|$ denotes the euclidean distance, and $\langle\cdot\rangle$ being the arithmetic mean over the respective trajectory; $\vec{r}_{i}^{D S}$ is the vector describing the geometric center of the $i$ th residue in the DS structure of ClCry4; the notation is chosen analogously for the RPD state. Equation (S1) describes the difference of the any-to-any distances of each residue for both the DS and the RPD simulation, averaged over time. The resulting matrix has dimension $N \times N$ with $N$ being the number of residues in the protein. 


\section{Principal Component Analysis}

Principal component analysis (PCA) was loosely following the guideline described in a separate study. ${ }^{2}$ The data set for the analysis is constructed using the geometric centers of every residue in the ClCry4 structure. Hence, the $i$ th residue supplies three numbers, $x_{i}, y_{i}$ and $z_{i}$, respectively. These three values for all residues are written into one $3 N$ dimensional vector $\vec{R}$. For ClCry4, the vector $\vec{R}$ will therefore contain $3 \cdot 498=1494$ elements. In order to achieve continious indexing for the vector $\vec{R}$, the indexes are shifted as follows:

$$
\vec{R}=\left(\begin{array}{c}
x_{1} \\
y_{1} \\
z_{1} \\
x_{2} \\
\vdots \\
x_{N} \\
y_{N} \\
z_{N}
\end{array}\right)=\left(\begin{array}{c}
R_{1} \\
R_{2} \\
R_{3} \\
R_{4} \\
\vdots \\
R_{3 N-2} \\
R_{3 N-1} \\
R_{3 N}
\end{array}\right) .
$$

The vector $\vec{R}$ can be written for every snapshot and for both the DS and the RPD state of ClCry4. $\vec{R}_{i}^{D S}$, for instance, would denote the vector for snapshot $i$ in the DS trajectory.

Starting from the $\vec{R}$ vectors, one can compute the $3 N \times 3 N$ co-variance matrix $C$ with entrees $c_{i j}$, defined as:

$$
c_{i j}=\left\langle\vec{R}_{i} \vec{R}_{j}\right\rangle-\left\langle\vec{R}_{i}\right\rangle\left\langle\vec{R}_{j}\right\rangle .
$$

Here, $\langle\cdot\rangle$ denotes the average over the trajectory for the merged DS and RPD state simulations. For instance, for the discussed analysis, $\mathcal{N}=125,000$ snapshots were considered, leading to 


$$
\left\langle\vec{R}_{i} \vec{R}_{j}\right\rangle=\frac{1}{2 \mathcal{N}}\left(\sum_{k=1}^{\mathcal{N}} \vec{R}_{i k}^{D S} \vec{R}_{j k}^{D S}+\sum_{k=1}^{\mathcal{N}} \vec{R}_{i k}^{R P D} \vec{R}_{j k}^{R P D}\right) .
$$

The square matrix $C$ is real and symmetric, allowing the calculation of its eigenvalues, which in turn are real and positive by the design of $C$. Furthermore, the eigenvalues and, therefore, the associated eigenvectors were sorted in decreasing order. The largest eigenvalue $\lambda_{1}$ is called the most dominant eigenvalue with the most dominant eigenvector $\overrightarrow{\Lambda_{1}}$. Another useful quantity associated with the principal components is the percentage of the total variance covered by that component defined as:

$$
\eta_{j}=\frac{\lambda_{j}}{\sum_{i=1}^{3 N} \lambda_{i}} .
$$

Looking at the principal components, one can distinguish regions in the protein reacting to changes caused by its transformation from DS to the RPD state. This can be seen by calculating

$$
R S_{i j}=\sqrt{\left(e_{j(3 i-2)}^{2}+e_{j(3 i-1)}^{2}+e_{j(3 i)}^{2}\right) \cdot \lambda_{j}}
$$

with $e_{j \xi}$ being the $\xi$ th entry in eigenvector $\overrightarrow{\Lambda_{j}}$ (jth principal component), while $i$ labels the residues in the protein. Equation (S6) yields the root square per residue of a principal component weighted by its respective eigenvalue $\lambda_{i}$.

As a second step one can consider the principal component's influence on the overall protein dynamics by working with configurations taken from individual snapshots. For further preparation, one normalizes the $\vec{R}$ vector describing each snapshot to the overall average of the jointed trajectories for the DS and RPD state. This overall average is expressed in a vector $\langle\vec{R}\rangle$ which is given by

$$
\langle\vec{R}\rangle=\frac{1}{2 \mathcal{N}}\left(\sum_{k=1}^{\mathcal{N}} \vec{R}_{k}^{D S}+\sum_{k=1}^{\mathcal{N}} \vec{R}_{k}^{R P D}\right),
$$


with $\mathcal{N}$ being the number of snapshots in the simulation as in Eq. (S4). This average is being used to calculate the vector

$$
\vec{\delta}^{D S}(k)=\vec{R}_{k}^{D S}-\langle\vec{R}\rangle
$$

and an analogous vector $\vec{\delta}^{R P D}(k)$ for the RPD state. One can now compare the projection, the similarity, of the vectors $\vec{\delta}^{D S}(k), \vec{\delta}^{R P D}(k)$ to the principal components. This is achieved by calculating the dot product, for instance, for the first principal component $\overrightarrow{\Lambda_{1}}$ as

$$
\begin{gathered}
\alpha^{D S}(k)=\vec{\Lambda}_{1} \cdot \vec{\delta}^{D S}(k) \\
\alpha^{R P D}(k)=\vec{\Lambda}_{1} \cdot \vec{\delta}^{R P D}(k)
\end{gathered}
$$

for all snapshots $k$. The time evolution of the resulting two values $\alpha^{D S}(k)$ and $\alpha^{R P D}(k)$ can then be plotted for each residue of the ClCly4 structure to realize a visualization of the differences between the DS and the RPD state within the protein structure associated with the corresponding principal component. 


\section{Supporting Results}

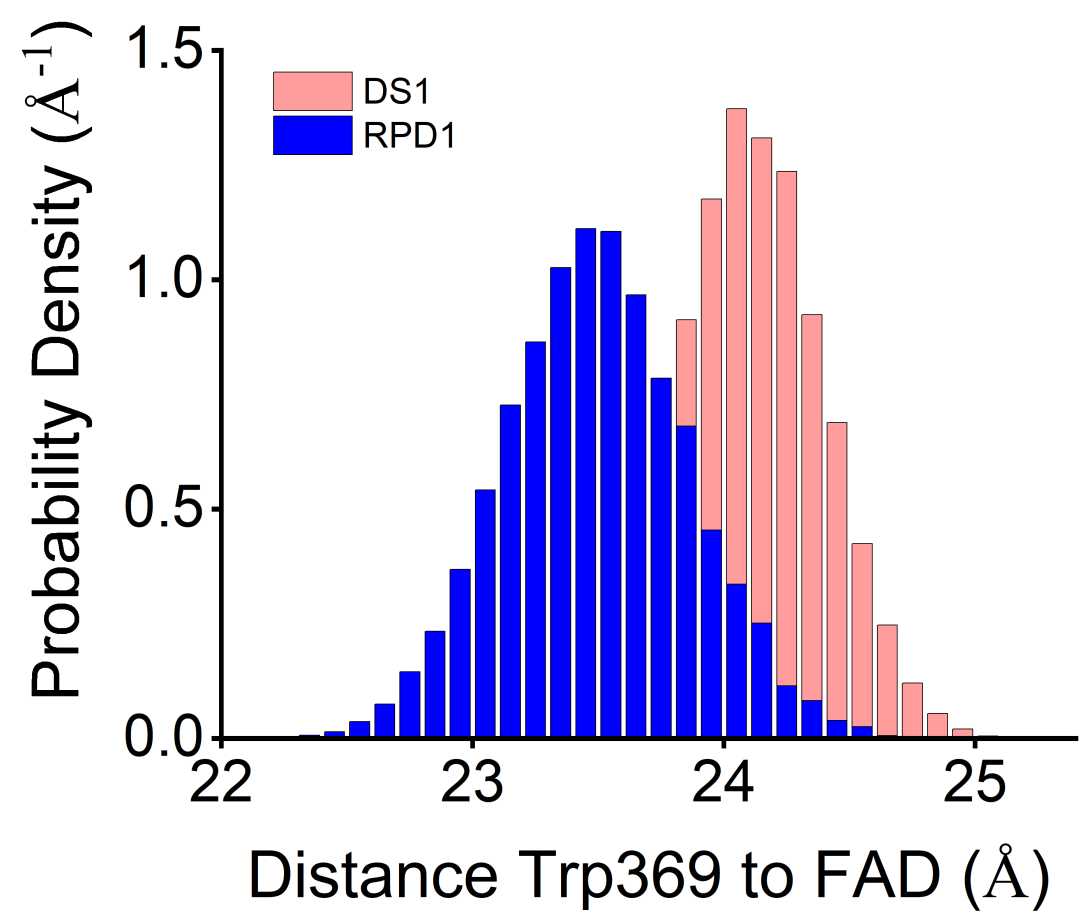

Figure S2: The probability density distribution of the center-to-center distance between Trp369 and FAD is shown for the DS1 and RPD1 simulations. One can see that Trp369 is on average closer to the FAD in the RPD1 state compared to DS1. Such a difference in distance could, for instance, lead to a significant change in electron transfer rate involved in ClCry4's activation process. 


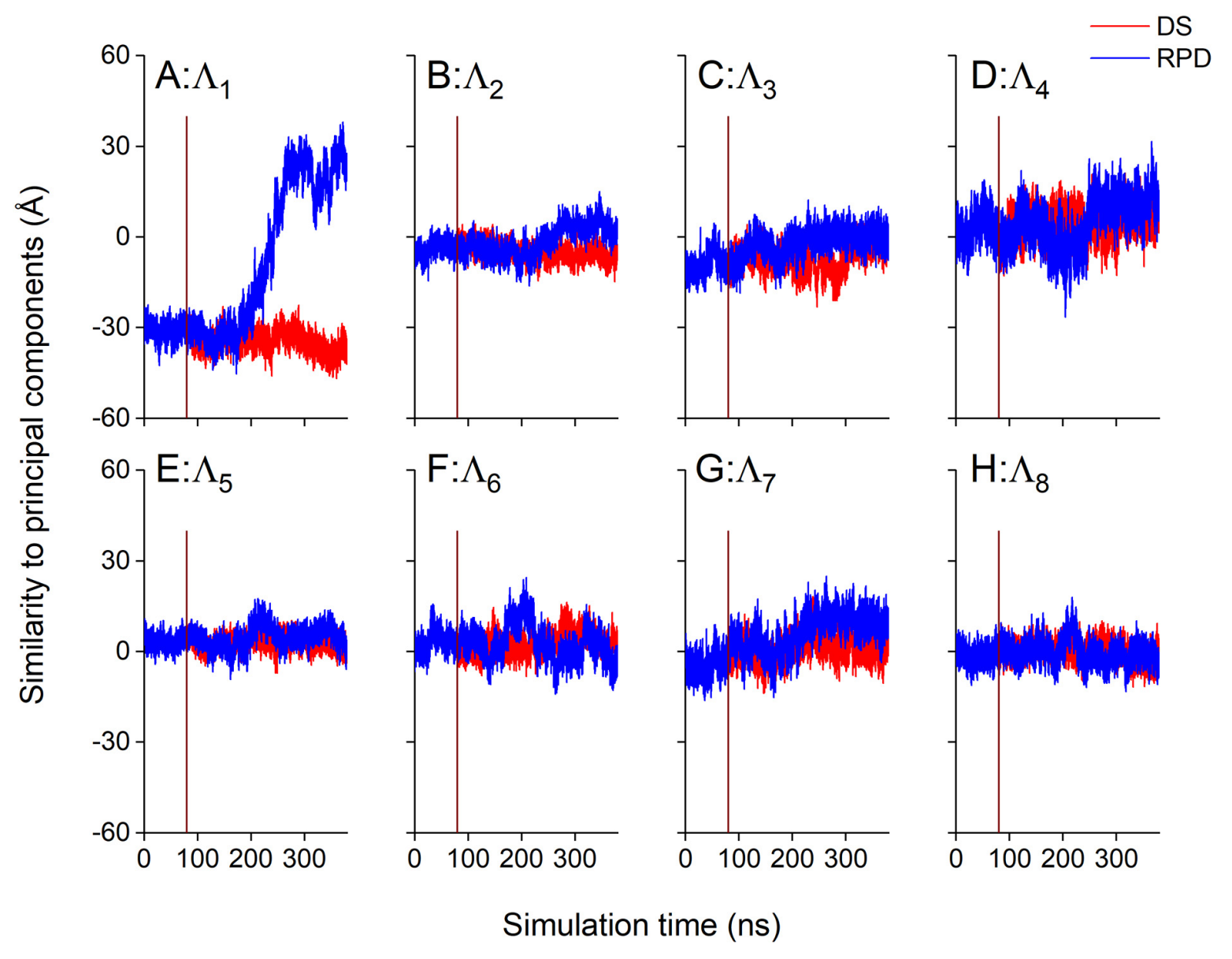

Figure S3: Fig. 5 showed a clear difference between the DS1 and RPD1 states of the ClCry4 protein. A relevant question is to investigate how quickly, after the creation of the RPD1 state, such a great difference becomes visible. Hence, the analysis, which was based on Eq. (S9) and Eq. (S10), was repeated for the simulation time interval $-80-300 \mathrm{~ns}$, corresponding to the time nomenclature shown in Fig. 1. The time of the creation of the RPD1 state is visualized by the vertical dark red line. Roughly 100 ns after the RPD1 state was initiated, the protein structure changes its conformation in respect to the first principal component as described in Panel A. The subsequent principal components exhibit almost no distinct changes. Only $\Lambda_{2}$ and $\Lambda_{7}$ show a minor increase. If, however, this increase is compared to the analysis in Fig. 5, the increase does not hold as the difference in mean values vanishes. 

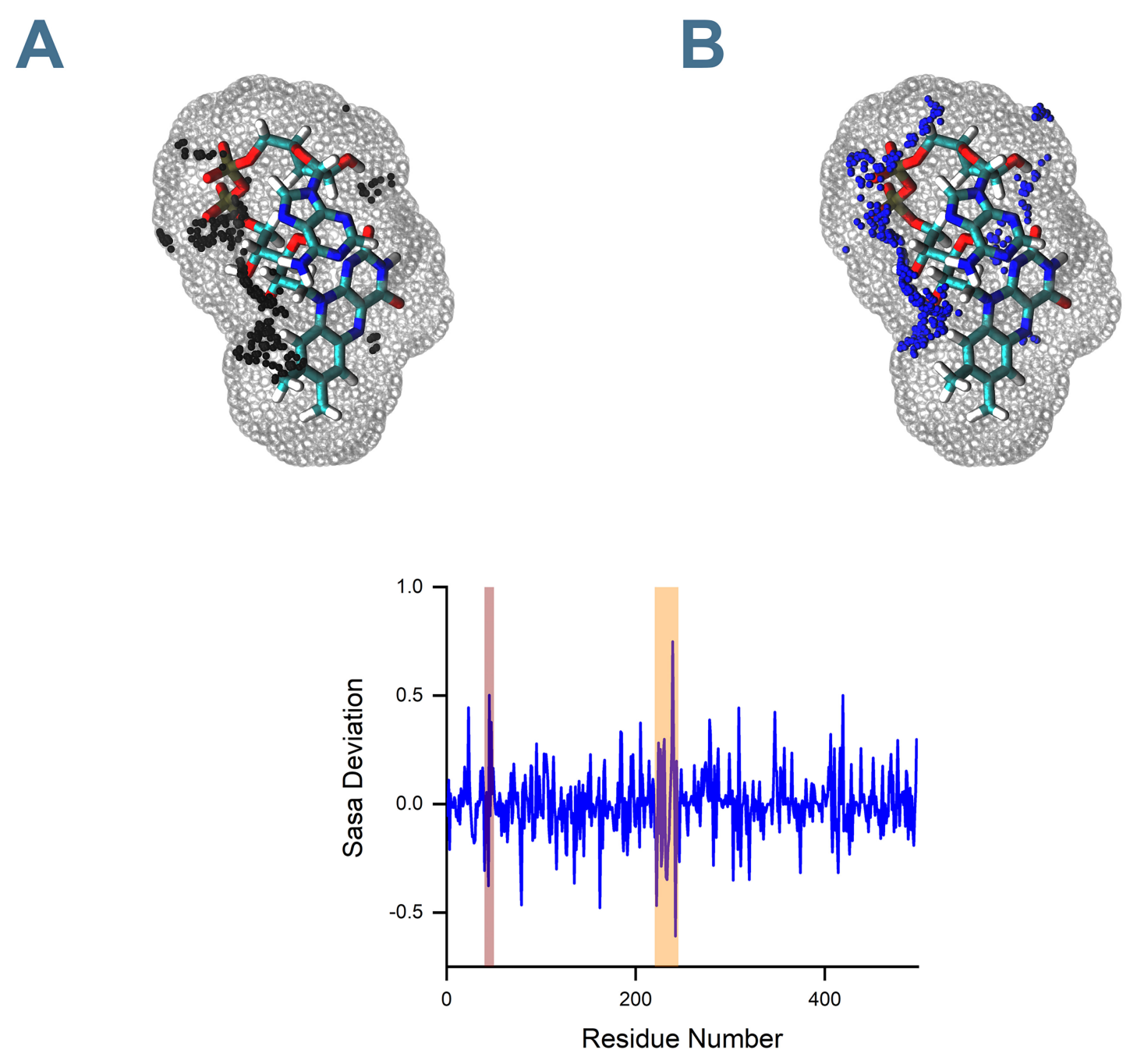

Figure S4: Panels A and B: The FAD cofactor is shown with the corresponding SASA representation for the DS1 (Panel A) and the RPD1 state (Panel B). For an isolated FAD in solution, the SASA is visualized by the gray spheres. As FAD is bound within the ClCry4 protein structure, its SASA is reduced. Panel A shows with black spheres the areas at which the solvent can reach the FAD in the DS1, while in Panel B, the blue spheres mark the areas that exhibit contact to the solvent in the RPD1 state. Visually, an increase in SASA can be clearly seen. Calculating the actual SASA values reveals an increase from $36.44 \AA^{2}$ to $60.38 \AA^{2}$. The representations were obtained using $\mathrm{VMD}^{3}$ and the SASA script used in a previous study. ${ }^{4}$ Panel $\mathrm{C}$ shows the normalized change in SASA upon the transition from the DS1 to the RPD1 state for each residue in ClCry4. The highlighted areas show the moving regions, residues 40-50 marked in red and residues 220-245 marked in orange. One can see that the SASA changes most notably in these regions as well. 
Table S1: Normalized solvent accessible surface area (SASA) values for all residues in ClCry4 (simulation set 1). The SASA is calculated with a sphere size of 1.4 and divided by the free SASA of each residue.

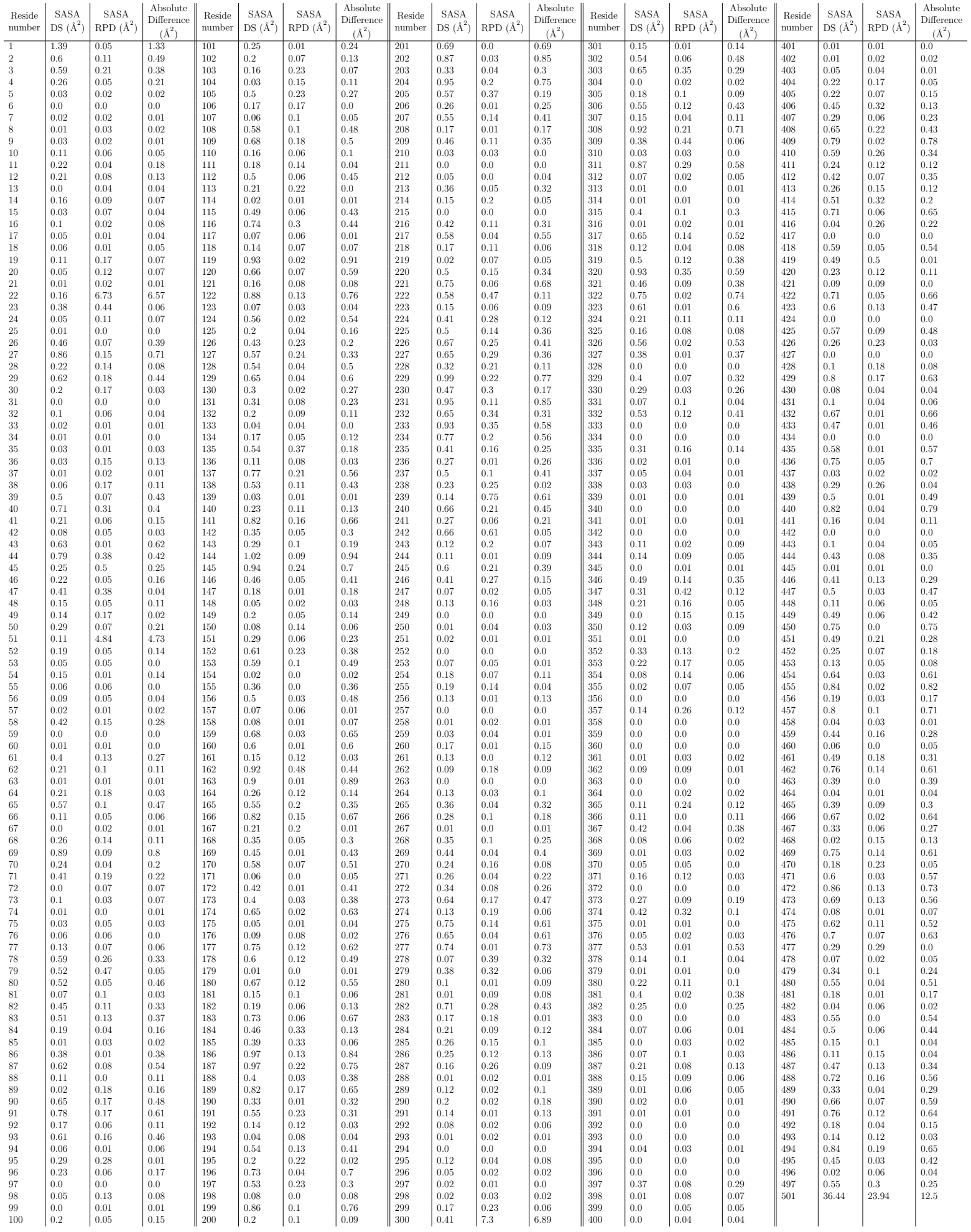




\section{References}

(1) Kattnig, D. R.; Nielsen, C.; Solov'yov, I. A. Molecular Dynamics Simulations Disclose Early Stages of the Photo-Activation of Cryptochrome 4. New J. Phys. 2018, 20, 083018.

(2) David, C. C.; Jacobs, D. J. Methods Mol. Biol.; Humana Press, Totowa, NJ, 2014; Vol. 1084; pp 193-226.

(3) Humphrey, W.; Dalke, A.; Schulten, K. VMD: Visual Molecular Dynamics. J. Mol. Graph. 1996, 14, 33-38.

(4) Schuhmann, F.; Korol, V.; Solov'yov, I. A. Introducing Pep McConst - A user-friendly peptide modeler for biophysical applications. J. Comput. Chem. 2021, 42, 572-580. 